\title{
SCIENTIFIC-EDUCATIONAL DISTRIBUTED LABORATORY - SOFTWARE AND HARDWARE MEANS
}

\author{
PRYANICHNIKOV, V[alentin]; ANDREEV, V[ictor]; BOBROV, P[avel]; BIRYUKOVA, E[lena]; \\ FROLOV, A[lexandr]; KHARIN, K[onstantin]; KIRSANOV, K[irill]; KOSTIN, A[leksei]; \\ KUVSHINOV, S[ergey]; MARZANOV, Y[urij] \& PRYSEV, E[vgeniy]
}

\begin{abstract}
Building a multi-unit mobile robot group acting as a single interrelated complex is one of the principal tasks of the development of efficient intellectual robotic technology. This task can be efficiently solved by creating a situation center and software and hardware means for the support of information, analytical and management activities. Creating intellectual educational systems for preparing specialists, as well as for autonomous "self-learning" of groups of interacting robots in the course of finding the solutions for placed tasks.

Keywords: mobile robots, remote control, networking technologies, videoconferencing, learning systems
\end{abstract}

\section{INTRODUCTION}

Cases are not uncommon when during the rectification of the consequences of emergency situations (ES) a necessity to perform the works by a robot group is arising. According to the researches, building a multiunit mobile robot group acting as a single interrelated complex is one of the principal tasks of the development of efficient intellectual robotic technology being able to operate in combat conditions, including the liquidation of major ES [1]. In a number of cases it is also necessary to receive timely consultations of specialists in various fields or to access a specific database. Creating intellectual educational systems for preparing specialists that work with information streams and robotic systems, as well as for autonomous "self-learning" of groups of interacting robots in the course of finding the solutions for placed tasks is by no means unimportant. The present paper is dedicated to the topical subject of building adequate information support of such works.

Such objectives can be placed in the field of space robotic systems as well. For example, the following technological tasks are accentuated in the paper [2]: development of group control methods for space-mission robotic systems, as well as for their information exchange; development of remote control methods for autonomous robotic systems on the basis of networking technologies, self-diagnosis and self-learning; establishing an advanced human-machine interface.

\section{SITUATION CENTER}

In both cases the task can be efficiently solved by creating a single situation center and software and hardware means for the support of information, analytical and management activities. Such statement of the problem outlines the creation of a hierarchical system for establishment and exchange of information and control data flows as the most natural way of solving it. This, in its turn, brings about a necessity to find a mechanism which enables solving the problem of interaction of groups of mobile robots (MR) and command posts simultaneously serviced by multiple operators, as well as the situation center as the top of the hierarchy. Establishing such communication channels that allow placing the unified situation management command center at a distance of many kilometers from the zone of works is a necessary condition of interaction.

It is also important to take into consideration the fact that the works are usually performed by Mobile Robots that should not be restricted in their movements. Furthermore, the bandwidth of communication channels should provide the transfer of multiple video streams from the computer vision systems of mobile robots both to the command posts and to the situation center. The solution of the said problem lies obviously in using network technologies. The essential fact is that the elements of the complex should serve as mobile nodes of the local area network (LAN). The structure of such complex is shown on fig. 1.

When such situation center is being created, it is reasonable to base on the experience of the Sensorika International Laboratory, which was accumulated in the course of development of virtual distributed laboratory [3]. It is designed for remote interaction, including the education of specialists [4]. Using networking technologies enabled consolidating several mobile robots, remote systems ("satellites"), repeaters and control consoles into a unified computing network. The Internet was used as the information transfer medium. VPN channels were used in order to provide protection from unauthorized access. A network of mobile robots for special and educational purposes was created in the building of the Institute of New Educational Technologies and IT Implementation of the Russian State University for the Humanities (RSUH), in the Keldysh Institute of Applied Mathematics of the Russian Academy of Sciences (KIAM), and in the Institute of Automation and Control Processes of the Siberian Branch of the Russian Academy of Sciences (DVGTU, in the city of Vladivostok). 


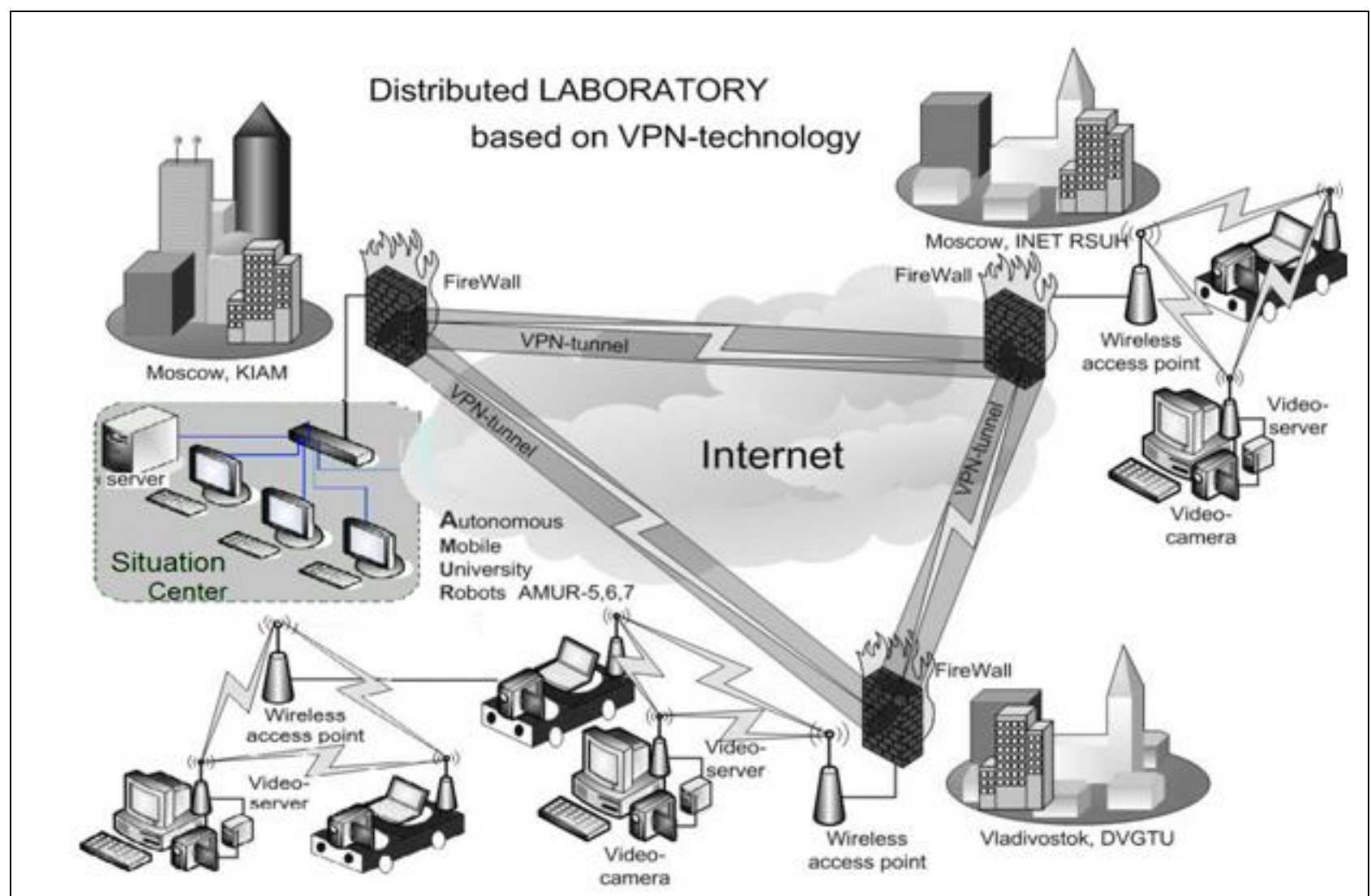

Fig. 1. Virtual scientific-educational distributed laboratory, which models the structure of information, analytical and management activities support complex

\section{DISTRIBUTED LABORATORY INFRASTRUCTURE}

The researches carried out on this structure gave ground to formulating the principles of building and functioning of scientific-educational situation centers, which widely use the existing robotic systems. Software, which allows to view remotely the "scene" and to control these robots according to the developed technology of distortion and delay mitigation in communication channels, was developed. The computing network was based on the TCP/IP protocol suite, due to the reason that this solution is widely spread in the present time, and shows good results. The networks on the basis of TCP/IP protocol suite have considerable scalability and possess various technical implementations, which enables consolidating various software and hardware modules of all the elements of the complex into a single network. In this case the task can be resolved to creation of a Local Area Network, the nodes of which comprise digital IP video cameras and computing devices installed on board of Mobile Robots, on remote modules and repeaters, on the one side, as well as the repeater on the operator console and the computers of the situation center, on the other side; each of them having its own IP address from the same network. Such system possesses the following characteristics. 1. Scalability - the capability of easily adding any kind of resources: video cameras, computers, radio channels, and mobile robots. 2. Distribution - the capability to consolidate the computing resources of the system elements. 3. Multi-user mode - the capability to receive information (including images from $\mathrm{MR}$ video cameras) on several operator consoles, including the consoles of the situation center. Therefore it is possible to analyze the operation of mobile robots and to generate control commands at any distance via remote connection on the Internet. 4. Using digital image processing systems - using a combination of special-purpose processors and universal-purpose computers, which enables constantly improving the processing of images and utilizing the digital signal filtration. 5. High quality of images - the capability to use high-resolution video cameras with high precision of color transfer. 6 . Functionality - the digital channel can be used not only to transmit video signal, but also for sending sound signals and control signals for both the computer vision systems (for example, to the swiveling and inclining platforms), and the MR actuating mechanisms. A significant distinctive feature of the technology is the capability of introducing modifications into software in emergency situations. Such modifications can be also introduced dynamically, i.e. in the course of operation of both the MR and the complex as a whole. 7. Multiple video stream support - the capability to simultaneously transmit the video signals from multiple video cameras without reducing the image quality, which can be achieved by means of using high efficiency image sequence compression algorithms. Consolidating computer vision modules through the radio channel can be implemented via using the Wi-Fi or Wi-Max standard. 8. High interference protection - in comparison with the analogue systems the digital channels possess higher interference protection. 9. Security - the capability to utilize the methods of protection of information from 
unauthorized access (for example, VPN channels and/or encryption, authentication).

As a result of the research on creation of virtual distributed laboratory, a mechanism for simultaneous controlling of the operation of multiple mobile robots has been developed. A three-level management system was built for the abovementioned case. The first level comprises the mobile robots themselves equipped with sensor systems (supersonic, computer vision, etc.) and actuating mechanisms. The second level comprises autonomous control systems for separate robots, and MR operators. Wi-Fi digital radio channel was used to provide robot mobility. The third level comprises the top level regulation systems controlled and modified by the commander, who is also coordinating the work of the operators. In this structure the commander views the entire scene and receives the video information from the computer vision systems of all MR and remote modules participating in the work. When building the computing network on the basis of TCP/IP protocol suite it is sufficient to include the computer of the commander into this network and provide the system components with corresponding software. One of system embodiments is implemented on the basis of the mechanism of Petri net, which enables synchronizing the operation of separate agents.

The software of electronic training unit class for education and training developed in Sensorika International Laboratory for the Engineering, Technical and Educational Robotic Center of the Federal Agency for Atomic Energy was taken as a basis for developing such software [5]. This class is designed to provide high quality of education and regular training of the operators of ground-based robot complexes and remotely controlled systems. The class was created on the basis of the RobSim software complex and comprised 8 PCs and one server, which constituted a Local Area Network. It comprised 7 working places for operator training, one working place for the instructor, and one working place combined with the server for the observer. The developed software enabled conducting simultaneous education and training of 7 mobile robot operators by one instructor. Besides specifying the network structure, the task of creation of the common interaction scene for up to seven robots controlled by seven operators was solved thereby.

The interaction of the software components is performed by means of classic messaging mechanism without utilizing the mechanism of parallel execution. In this case user command processing, data transfer in the network and other operations are not carried out before the current cycle of message processing is completed. Modifying the cycle of message processing allowed reducing considerably the time of response of the robot model to the control commands sent by a remote operator $[6,7]$. The structure allows establishing connection to the Internet global network at its any point, i.e. anywhere where the network is accessible.

Controlling separate Mobile Robots that are the basic constituents of the distributed laboratory and onboard manipulators that are being developed is based on the new principles of the so-called "bionic control". Such an approach can be called biologically adequate control, and two essential know how should be pointed out when describing it: 1) control is based on natural synergism (on the basis of the characteristic vectors of the linearised Lagrange equations) that allows to "unleash" the degrees of freedom; 2) the model of controlling the articular momentum is implemented by using a visco-elastic spring with the feedback from the articular angle with a time delay. Such control provides "softness" and adaptability. For medical applications of such manipulator (an embodiment of the exoskeleton helping the patients to recover due to performing training movements is available) it is crucial that the parameters of the exoskeleton structure (the axes of rotation) and its control parameters (stiffness, viscosity) can be configured depending on the patients' individual parameters, which can be determined by analyzing the movement registration data. The corresponding system of data registration and analysis was developed and was subject to comprehensive approbation.

\section{VIDEOCONFERENCING TECHNOLOGY}

Another distinctive feature of the virtual laboratory is the wide usage of the videoconferencing technology. It is an efficient means for education as well as for joint practical work of operators, Mobile Robots and the commander or the instructor. A multi-user distributed videoconference on the basis of the Adobe Connect software was organized as an example. Among the participants of the videoconference there were several operators and two Robotino serial mobile robots manufactured by FESTO corporation equipped with web cameras. Additional cameras were also installed to provide a general overview of the zone of robot activities in the process of fulfilling the set objective (in practice such a camera can be installed on a separate robot and its task would be providing a maximum overview of the zone of works). With such organization of work the robot operators can additionally coordinate their actions with the purpose of fulfilling the objective more efficiently.

When such a system is used at the stage of developing the control software for MR, testing or education, the possibility of jointly elaborating the program code, the set of commands or configuring the equipment during a videoconference is by no means unimportant. Thereby the modifications introduced by any participant can be checked on-the-scene, their results and efficiency can be assessed. Such approach is also supported by utilizing the technologies of development of control programs for mobile robots on the basis of interpreters.

\section{LEARNING SYSTEMS}

During the process of creation of virtual distributed laboratory considerable attention was paid to the development of educational systems and environments that include various robotic devices. A formal model 
based on the system of interacting automatons, which is described in paper [8] and shown on the fig. 2, was taken as a basis for the education process.

In this model the education process is displayed as two interacting automaton models - the controlled automaton $\mathrm{A}_{0}$ (student) and the controlling automaton $\mathrm{A}$ (learning system / teacher). The training material, such as exercises, explanations, reference materials, etc., is the output $\mathrm{D}$ of the automaton $\mathrm{A}$, and, therefore, is the input of $\mathrm{A}_{0}$. The events that took place in the process of education (the answers of the student, the data on the usage of dictionaries, training material processing etc.) are the output $\mathrm{B}$ of the automaton $\mathrm{A}_{0}$, and, therefore, are the input of $\mathrm{A}$. The task of the educational automaton $\mathrm{A}$ is to control the automaton $\mathrm{A}_{0}$ by using the output $\mathrm{D}$ in such a manner that it reaches the required state in shortest time possible. Another variant of the control task may be achieving the maximum result in specified time.



Fig. 2. Automaton-based education process model

Based on the principles of such model, an interactive educational platform for teaching reading and comprehension of texts in foreign languages named LesenHQ [9] has been developed in cooperation with the Department of Slavic Studies of the Ruhr University. The development process was based on the following didactical principles: individualization, cooperative forms of work and education, orientation on apprehension and local knowledge networks, transparency and monitoring of education objectives, accounting the reading styles and strategies, exercise typology, journaling and differentiated grading, and additional information support. Vast amount of statistical information on student behavior in such educational environments was accumulated during the approbation of the courses of Russian and German languages developed for this platform. This information became the ground for building the universal algorithmic models of various components of the abovementioned automaton model (see $\mathrm{A}_{1}-\mathrm{A}_{5}$ on fig. 2), and for creating the automated intellectual educational systems. In particular, sets of rules for expert systems allowing to analyze the student behavior, his learning progress, choice of specific training materials or selection of global education strategy were developed.

Using robotic systems, including mobile robots, is possible on various levels of the proposed model. Thereby these systems may both play the additional part of "visual aid" during operator education, and implement the virtually autonomous education process during the interaction of groups of robots for solving complex tasks in the conditions of limited communication with the operator or the situation center. When the abovementioned approach is used, the decision making process at the situation center level, which takes place using the data from mobile robotic systems processed by distributed local control centers, becomes more adequate and efficient.

The present work was partially supported by the grant of the Russian Foundation for Basic Research №10-0700612 and 11-04-12073-ofi-m-2012.

\section{REFERENCES}

[1] Minakov E.P., Lopota V.A., Yurevich E.I., Kondratev A.S. Концепция развития робототехнических систем в интересах пилотируемой космонавтики, исследования Луны и планет солнечной системы. Экстремальная робототехника. Наномикро- и макророботы (ЭР-2009) // Maтериаль XX Международной научно-технической конференции. Таганрог: Изд-во ТТИ ЮФУ, 2009. - 390с. С.103 - 106

[2] Andreev V.P., Kirsanov K.B., Levinsky B.M., Pryanichnikov V.E. Проблемы построения сети интернет-лабораторий с использованием автономных мобильных учебных роботов "АМУР" // Информационно-измерительные и управляющие системьl, М.: Радиотехника, 2009. -Т.7, №6. - С.124 - 131

[3] Kuvshinov S.V.. Использование современных IT технологий для создания интерактивной образовательной среды с применением динамических трехмерных моделей // Интеллектуальные и адаптивные роботы, № 1-2, 2009. с.9396

[4] Andreev V.P., Kirsanov K.B., Levinsky B.M., Pryanichnikov V.E., Travushkin A.S. Создание класса электронных тренажеров на основе быстродействующей локальной вычислительной сети // Информационно-измерительные $u$ управляюшие системы, М.: Радиотехника, 2009. -Т.7, №6. C.82-92

[5] Stopper, M. \& Katalinic, B.: Service-oriented Architecture Design Aspects of OPC UA for Industrial Applications, Proceedings of the International Multi-Conference of Engineers and Computer Scientists 2009, Vol II, ISBN: 978-988-17012-7-5, IMESC 2009, March 18 - 20, 2009, Hong Kong

[6] Andreev V.P., Pryanichnikov V.E. \& Prysev E. A. Multi-Access Control of Distributed Mobile Robotic Systems Based on Networking Technologies // Annals of DAAAM for 2010 \& Proceedings of the 21st International DAAAM Symposium, Editor B. Katalinic, Published by DAAAM International, Vienna, Austria 2010, pp. 15 - 16

[7] Kudryavcev V.B., Aliseychik P.A., Waschik K., Knap Zh., Strogalov A.S., Shekhovcov S.G. Моделирование процесса обучения // Интеллектуальные системы. Т. 10, вып. 1-4. 2006. C. 189-271

[8] Waschik K., Kharin K. LesenHQ - Kompetenz in Fremdsprachen - didaktisches Outline. LesenHQ - Competency in Foreign Languages - Didactic Outline. Available from: http://www.lesenhq.net/pdf/lesenhq_didaktisches_outline2.pdf Accessed: 2012-07-23 